\title{
NONRECURSIVE ALGORITHM FOR REMOTE GEOLOCATION USING RANGING MEASUREMENTS
}

\author{
P. L. KAUFMANN, R. VILHENA DE MORAES, H. K. KUGA, L. A. BERALDO, \\ C. N. MOTTA MARINS, AND P. KAUFMANN \\ Received 18 April 2006; Revised 11 August 2006; Accepted 25 September 2006
}

The objective of this work is to present a new algebraic solution for the problem of remote determination of geographic coordinates of a target, using a new remote geopositioning system being developed in Brazil. It can be useful for double-check measurements obtained with other methods, for certain critical applications, being capable to perform independently from them. This system requires three-reference bases on the surface of the earth with synchronized clocks and a repeater in space. Calculations are derived from measurements of propagation time of clock signal transmitted by one base to all bases and target, via a transponder in space. The algorithm also provides the "instantaneous" determination of the repeater position in space and brings other applications in navigation and remote clock synchronization. The algorithm has been successfully tested through computational software.

Copyright (c) 2006 P. L. Kaufmann et al. This is an open access article distributed under the Creative Commons Attribution License, which permits unrestricted use, distribution, and reproduction in any medium, provided the original work is properly cited.

\section{Introduction}

The first space technique used for remote geopositioning and navigation was based on Doppler effect measured on transmitted signals from and to low orbiting satellite $[3,6]$. The technique remains in use by various services. As a satellite moves with respect to a target, each measured Doppler frequency shift determines a half-cone with the satellite at its apex, with the velocity vector as its axis of symmetry. The half-cone angle depends on the satellite velocity, the frequency transmitted, and the Doppler shifted frequency. Similar curves are obtained from reference bases at known positions. The inflection point of the slope of a Doppler curve obtained as the satellite flies over a transmitting target determines the distance to the satellite. Iterative last-squares procedure is used to calculate the actual location of the target, which often requires several satellite passes (or several satellites at a time) $[1,3,6]$. 
Most of positioning, navigation, and time synchronization are currently achieved using the global positioning system (GPS), based on time data transmitted from slow moving high altitude orbiting satellites, whose positions in the sky are known and constantly updated $[2,10]$. GLONASS and GALILEO are other systems based on the same GPS concept. The target receives precise time signals from the system satellites in the sky, whose positions need to be constantly updated. Distances from the target to the satellites are obtained from time differences measured at the target, referred to its own clock, which is not necessarily synchronized to the satellites' clocks. The basic requirement to obtain the receiver clock error is that there are four satellites visible at a given epoch. With more than four satellites visible, more accurate determinations are obtained by recursive procedures.

A new concept for remote geopositioning, time dissemination, and navigation is currently being developed in Brazil (see $[4,5,8]$ ) to which the present algorithm is addressed. The system requires three reference bases on the ground, with well-known geodetic positions, carrying synchronized clocks, a transmitter in one base emitting time coded marks, and one repeater in the sky (Figure 1.1). Base $A$ transmits the clock information to the repeater in the sky that retransmits it to all bases, including base $A$ and the target. The arrival times at the bases and target are compared to the clock mark sent by base $A$, producing the time differences that are used in the presented algorithm. With a single coded clock transmission it is possible to determine the distances from the stations to the repeater and its coordinates in the sky. The target coordinates are determined from several measurements, in succession with a moving repeater, or simultaneously if multiple repeaters are available. Since the distance determinations are based on propagation times, the essential requirement is the knowledge of delays caused by cables, components, circuits, and system loops at the bases, the target, and at the sky transponder, which must be determined carefully to proceed with the calculations.

The present algorithm is addressed to three principal applications:

(a) trajectory determination of a moving platform carrying the repeater, satellite orbitography when carried by a spacecraft;

(b) target geopositioning;

(c) time synchronization transfers to other sites with well-known geodetic positions.

Applications $a$ and $b$ will be considered in Section 2. Application $c$ is discussed in Section 3.

The implementation of the new positioning system becomes particularly important to allow independent determinations to compare and double-check measurements taken by other systems (GPS, Doppler-Argos). This is needed in certain critical applications such as the safe repeatable positioning of underwater obstacles endangering ship navigation, or recovery of false measurements caused by severe geomagnetic storms interferences on other systems. This new system, designated as regional positioning system (RPS) or geolocal, is applicable over regional areas which extensions depend on the altitude of the repeater(s) with respect to the bases and targets. It might cover tens to hundred $\mathrm{km}$ for a repeater(s) in low flying balloons or aircrafts to thousands $\mathrm{km}$ for a repeater in satellite. The RPS has potential to become less sensitive compared to other systems to interferences on spacecraft hardware and transmissions caused by severe space weather disturbances. 


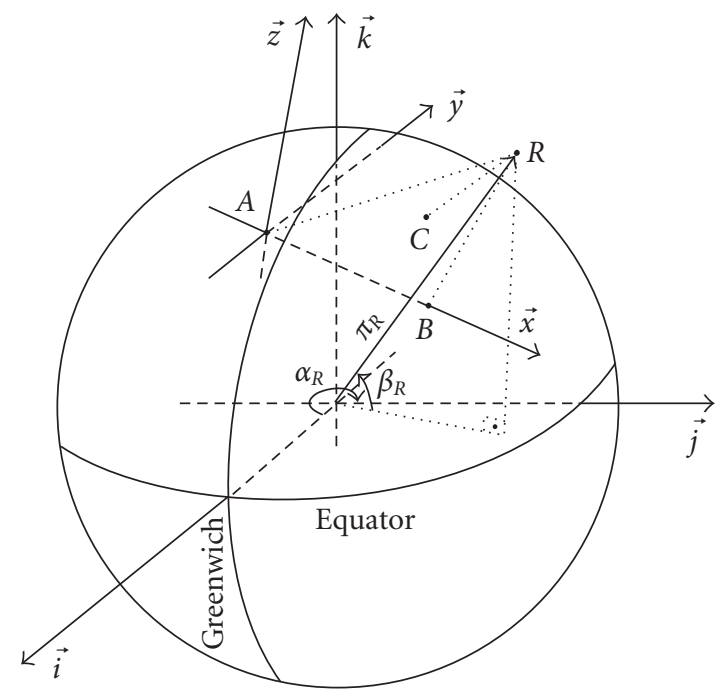

Figure 1.1. Spherical and Euclidean coordinate systems for the reference bases, $A, B$, and $C$, and repeater, $R$.

\section{Repeater and target positions determination}

The determination of the target coordinates is done by time difference measurements obtained at the bases and at the target, in succession if the repeater is carried by a moving platform (such as a satellite), or with fewer time interactions when there are multiple repeaters above the target's horizon. One necessary condition is that the clocks at the bases and the target are synchronized. It is to be reminded that the repeater's position with respect to the fixed bases on the ground is determined with a single time difference interaction, or a single "snapshot," irrespectively from the nature or the movement of the platform carrying the repeater. Such position is determined a single time, and does not require any correction (such as relativistic corrections).

The first measurement is done as follows: base $A$ sends a time signal at a given instant, kept as reference. The time signal arrives at the repeater and is retransmitted to the bases $A, B, C$ and to the target $P$, which copy the instants of arrival. The time differences at $A, B, C$, and $P$ referred to the instant the time signal was transmitted by base $A$, are sent to a processing center, which may be base $A$, for example. We obtain the following time differences: $\Delta_{1} t_{A}, \Delta_{1} t_{B}, \Delta_{1} t_{C}$, and $\Delta_{1} t_{P}$, respectively. The process is repeated three more times, successively, providing $\Delta_{2} t_{A}, \Delta_{2} t_{B}, \Delta_{2} t_{C}$, and $\Delta_{2} t_{P}, \Delta_{3} t_{A}, \Delta_{3} t_{B}, \Delta_{3} t_{C}$, and $\Delta_{3} t_{P}$, and $\Delta_{4} t_{A}, \Delta_{4} t_{B}, \Delta_{4} t_{C}$, and $\Delta_{4} t_{P}$. These $\Delta t$ 's include the transmission delays due to cables, electronics, calculation cycles, and so forth, from transmission by base $A$, retransmission by the repeater in the sky, and reception at bases $A, B, C$, and target.

On a more general standpoint the solution of the problem is developed as follows: for each one of the four time difference set of measurements (e.g., $\Delta_{1} t_{A}, \Delta_{1} t_{B}, \Delta_{1} t_{C}$, and $\Delta_{1} t_{P}$ 
as the first set of measurements), it is possible to determine the repeater's position at a given instant and the distance from the target to the repeater at that same instant. In other words, a sphere is determined in space, centered at the repeater's position, with radius equal to the distance of the repeater to the target. Therefore, after all time differences measurements are made, we have four spheres determined in space, which intercept each other at a single point, the target position. The algebraic solution for the determination of this point is obtained according to the following steps.

Considering the first set of measurements we will determine the repeater's position $R_{1}$ and the corresponding distance to the target, $P R_{1} . P R_{1}$ as well as $A R_{1}, B R_{1}$, and $C R_{1}$ (resp., the distances of the bases $A, B$, and $C$ to $R_{1}$ ) are obtained solving the linear system

$$
\begin{gathered}
\Delta_{1} t_{A}=\frac{2 A R}{c}+\delta_{A_{t}}+\delta_{R}+\delta_{A_{r}}, \\
\Delta_{1} t_{B}=\frac{A R}{c}+\frac{B R}{c}+\delta_{A_{t}}+\delta_{R}+\delta_{B_{r}}, \\
\Delta_{1} t_{C}=\frac{A R}{c}+\frac{C R}{c}+\delta_{A_{t}}+\delta_{R}+\delta_{C_{r}}, \\
\Delta_{1} t_{P}=\frac{A R}{c}+\frac{P R}{c}+\delta_{A_{t}}+\delta_{R}+\delta_{P_{r}},
\end{gathered}
$$

where the measured instrumental time delays are: at the transmitter, $\delta_{A_{t}}$, at the transponder, $\delta_{R}$, and at the bases and target receivers, $\delta_{A_{r}}, \delta_{B_{r}}, \delta_{C_{r}}$, and $\delta_{P_{r}}$, respectively.

We still need to calculate $R_{1} . R_{1}$ is in the intersection of the spheres centered in $A, B$, and $C$ with radii $A R_{1}, B R_{1}$, and $C R_{1}$, respectively. It is to be noted that the intersection of these three spheres determines two points in space; one of them may be disregarded because it should be located internally to the earth.

In order to simplify the description of these spheres centered in $A, B$, and $C$, it is convenient to describe them in an orthonormal coordinates system $(A, \vec{x}, \vec{y}, \vec{z})$ centered in $A$, with $B$ contained by the straight line determined by $A$ and $\vec{x}, C$ contained by the plane determined by $A, B$, and $\vec{y}$, and with $\vec{z}$ pointing away from the earth in $A$. It is easy to achieve algorithmically this operation when $A, B$, and $C$ are relatively close to each other (e.g., in the same octant) and under the condition that they are not aligned. Let us denote by

$$
M:\left[0,360^{\circ}\left[\times\left[-90^{\circ}, 90^{\circ}\right] \times\left[0, \infty\left[\longrightarrow \mathbb{R}^{3}\right.\right.\right.\right.
$$

the function that has as variables the longitude, latitude, and the distance of a given point to the center of the earth, respectively, and as values the coordinates for that same point in $(A, \vec{x}, \vec{y}, \vec{z})$. 
The equations of the spheres centered in $A, B$, and $C$, with respective radii $A R_{1}, B R_{1}$, and $C R_{1}$ in the system $(A, \vec{x}, \vec{y}, \vec{z})$ simply become

$$
\begin{gathered}
x_{R_{1}}^{2}+y_{R_{1}}^{2}+z_{R_{1}}^{2}=A R_{1}^{2}, \\
\left(x_{R_{1}}-A B\right)^{2}+y_{R_{1}}^{2}+z_{R_{1}}^{2}=B R_{1}^{2}, \\
\left(x_{R_{1}}-x_{C}\right)^{2}+\left(y_{R_{1}}-y_{C}\right)^{2}+z_{R_{1}}^{2}=C R_{1}^{2},
\end{gathered}
$$

where $x_{C}=\langle\overrightarrow{A C}, \vec{x}\rangle$ and $y_{C}=\langle\overrightarrow{A C}, \vec{y}\rangle$.

The solution of the system above is

$$
\begin{gathered}
x_{R_{1}}=\frac{A R_{1}^{2}-B R_{1}^{2}+A B^{2}}{2 A B}, \\
y_{R_{1}}=\frac{r_{1}^{2}-r_{2}^{2}}{2 y_{C}}+\frac{y_{C}}{2}, \\
z_{R_{1}}=\sqrt{r_{1}^{2}-y_{R_{1}}^{2}},
\end{gathered}
$$

where $r_{1}^{2}=A R_{1}^{2}-x_{R_{1}}^{2}$ and $r_{2}^{2}=C R_{1}^{2}-\left(x_{C}-x_{R_{1}}\right)^{2}$.

Observe that by putting $z_{R_{1}}=-\sqrt{r_{1}^{2}-y_{R_{1}}^{2}}$ we also obtain a solution for the system (2.3), but this one is disregarded because a point with negative coordinate $z$ is located inside the earth (justifying the care that must be taken so that $\vec{z}$ points away from the earth).

If latitude, longitude, and altitude of the repeater are needed, they can already be obtained by computing $M^{-1}\left(x_{R_{1}}, x_{R_{1}}, x_{R_{1}}\right)$.

By repeating the process described above we obtain the equations in the system $(A, \vec{x}$, $\vec{y}, \vec{z})$ for the four spheres whose intersection will be the target position as desired.

Similarly, as we did by establishing the system $(A, \vec{x}, \vec{y}, \vec{z})$ as a function of the points $A, B$, and $C$, we will establish a system $\left(R_{1}, \vec{u}, \vec{v}, \vec{w}\right)$ as a function of the points $R_{1}, R_{2}$, and $R_{3}$ (see Figure 2.1). As in the previous case, we get one orthonormal system, where $R_{2}$ is contained by the straight line determined by $R_{1}$ and $\vec{u}$, and $R_{3}$ is contained by the plane determined by $R_{1}, R_{2}$, and $\vec{v}$.

Similarly, the coordinates $\vec{u}, \vec{v}$, and $\vec{w}$ of the target position, denoted by $P$, form a solution for the system

$$
\begin{gathered}
u_{P}^{2}+v_{P}^{2}+w_{P}^{2}=P R_{1}^{2}, \\
\left(u_{P}-R_{1} R_{2}\right)^{2}+v_{P}^{2}+w_{P}^{2}=P R_{2}^{2}, \\
\left(u_{P}-u_{R_{3}}\right)^{2}+\left(v_{P}-v_{R_{3}}\right)^{2}+w_{P}^{2}=P R_{3}^{2},
\end{gathered}
$$

where $u_{R_{3}}=\left\langle\overrightarrow{R_{1} R_{3}}, \vec{u}\right\rangle$ and $v_{R_{3}}=\left\langle\overrightarrow{R_{1} R_{3}}, \vec{v}\right\rangle$. 


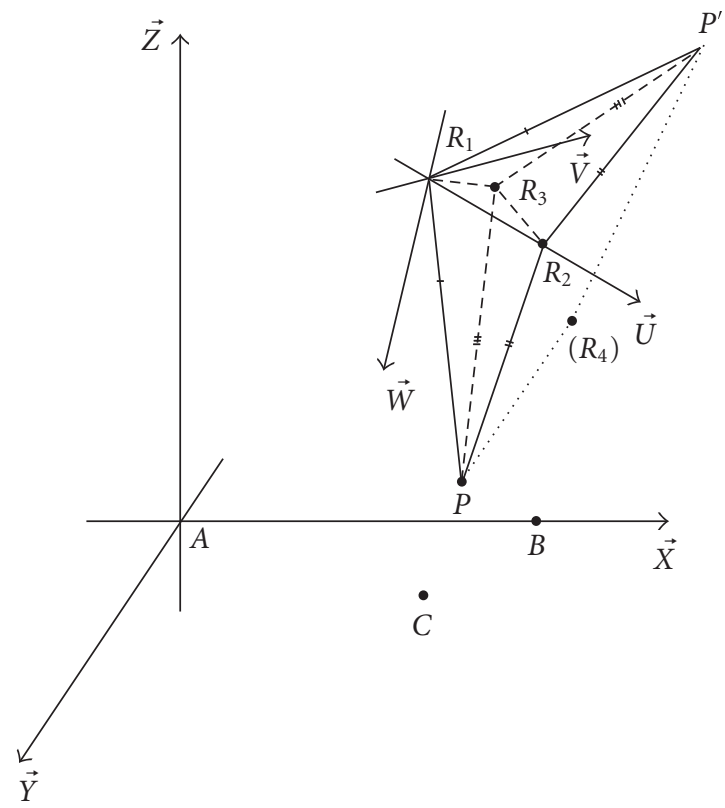

Figure 2.1. Four repeater positions $R_{1}, R_{2}, R_{3}$, and $R_{4}$, and possible target positions, $P$ and $P^{\prime}$.

This system admits two points as solutions

$$
\begin{gathered}
u_{P}=\frac{P R_{1}^{2}-P R_{2}^{2}+R_{1} R_{2}^{2}}{2 R_{1} R_{2}}, \\
v_{P}=\frac{\rho_{1}^{2}-\rho_{2}^{2}}{2 v_{R_{3}}}+\frac{v_{R_{3}}}{2}, \\
w_{P}= \pm \sqrt{\rho_{1}^{2}-v_{P}^{2}},
\end{gathered}
$$

where $\rho_{1}^{2}=P R_{1}^{2}-u_{P}^{2}$ and $\rho_{2}^{2}=P R_{3}^{2}-\left(u_{R_{3}}-u_{P}\right)^{2}$.

Here we cannot neglect the possibility of a negative value for coordinate $w_{P}$; the fourth sphere is required to decide if we take $w_{P}$ positive or negative. The two solutions can be then written in the system $(A, \vec{x}, \vec{y}, \vec{z})$ as $u_{P} \vec{u}+v_{P} \vec{v}+\sqrt{\rho_{1}^{2}-v_{P}^{2}} \vec{w}+R_{1}$ and $u_{P} \vec{u}+$ $v_{P} \vec{v}-\sqrt{\rho_{1}^{2}-v_{P}^{2}} \vec{w}+R_{1}$. To decide which one is the target position, we simply calculate the distance of each to $R_{4}$ and $P=\left(x_{P}, y_{P}, z_{P}\right)$ will be the one which has $P R_{4}$ as such distance.

Finally, we obtain the longitude, latitude, and altitude of $P$ computing $M^{-1}\left(x_{P}, y_{P}, z_{P}\right)$. 


\section{Remote clock synchronization}

Another important application of the algorithm above is the synchronization of clocks in remote known positions. The problem can be put in this way: given three bases on the ground with well-known positions and synchronized clocks, and a repeater in the sky, we wish to synchronize a clock at a known position with the other three. Let us denote the positions of the three bases by $A, B$, and $C$, and the position of the target whose clock we wish to synchronize by $P$.

First, we obtain a set of measurements like those described in the beginning of the previous section and obtain $\Delta t_{A}, \Delta t_{B}, \Delta t_{C}$, and $\Delta t_{P}$. Note that $\Delta t_{P}$ contains precisely the error we wish to determine in order to synchronize the clock at $P$.

The algorithm described in the previous section allows us to determine the position of the repeater on a given instant. Let us denote this position by $R$. As the position of the target is known, we can compute the theoretical value for $\Delta_{P}$, that will be denoted by $\Delta_{P}^{\prime}$ as follows:

$$
\Delta_{P}^{\prime}=\frac{A R}{c}+\frac{P R}{c}+\delta_{A_{t}}+\delta_{R}+\delta_{P_{r}} .
$$

Therefore, the clock at $P$ can be synchronized simply by adding $\Delta_{P}^{\prime}-\Delta_{P}$ to it's current time.

\section{Final remarks}

The present study is intended to describe the method which will be used in future experimental applications. The algorithm has been successfully applied in computational software simulations using actual geographic locations and repeaters at various altitudes. A critical source of errors is related to the accuracy of the delays, measured experimentally. Simulations of uncertainties error propagation are being studied separately. It is expected that with the use of more than one repeater in the sky such effects will become considerably reduced.

The electronic delays of electronic subsystems are known within few nanoseconds, which is much smaller than the requirement of the proposed application, that aims moderate accuracies, better than about $10 \mathrm{~m}$ (or about 30 nanoseconds time in radio propagation). For radio transmissions in a frequency in the UHF range or higher, the terrestrial ionosphere refraction index effect in the signal propagation velocity may bring estimated uncertainties of the order of $0.1 \mathrm{~m}(0.3 \mathrm{~ns})$, which are negligible for the present application.

Studies are currently being developed extending applications to low earth orbits [9]. One experimental demonstration setup is currently being developed [7]. The test system consists in a digital clock coupled to a radio system. A reference base responsible for the time dissemination will transmit and receive the clock marks, while the others will only receive. All bases and targets record the times the transmitted clock mark was received, which are sent to the algorithm processing terminal. The clocks used in the experimental demonstration are being built using FPGA (flexible programmable gate array) chip technology, providing time accuracy of 1 nanosecond, which corresponds to an uncertainty of about $0.3 \mathrm{~m} \ll 10 \mathrm{~m}$ initially set for this application. Clocks are referred to thermally 
stable quartz oscillators, with short term stability of the order of one over $10^{11}$ of a second. Careful attention is being given to the instrumental delays (hardware, antennas, cables, instructions cycles). The time delays on the radios will be measured using logic analyzers and high speed oscilloscopes. Delays in transmission lines, antennas, and connectors will be measured using vector analyzers. Measured delays at transmission, repeater, and receptions are made available to the processing center. Clock synchronization will be accomplished by direct comparison at the bases and target, to a mobile master clock, with the same 1 nanosecond accuracy, repeated as many times to comply with the stability required by the experiments

Initially, the algorithm processing will be done at a reference base. Various well-known data transmissions means may be used to bring the measured time differences to the central processing center. For the first experimental demonstration we have considered the use of regular mobile telephone network. There are plans to install the algorithm at a low level language in the digital signal processor board used at all bases and targets.

\section{Acknowledgments}

We acknowledge one referee comments which were helpful to improve the quality of this presentation. This research is partially supported by the following Brazilian funding agencies: (a) Contract 01.05.0034.00 with FINEP_-Financiadora de Projetos of the Ministry of Science and Technology; (b) Grants 300141/02-5, 303143/04-5, and 304822/89-2 with the Conselho Nacional de Desenvolvimentos Científico e Tecnológico, CNPq.

\section{References}

[1] ARGOS, User's Guide-Satellite Based Data Collection and Location System, Service Argos, Toulouse, 1978.

[2] B. K. Cariveau and K. L. Therkelsen, Satellite data management in DoD NAVSTAR GPS receivers, Proceedings of IEEE Position Location and Navigation Symposium (PLANS '88), Florida, 1988, pp. 134-144, reprinted from Navigation: Land, Sea, Air \& Space (M. Kayton, ed.), IEEE Press, Selected Reprint Series, IEEE Aerospace and Electronic System Society, New York, 1990, pp. $120-130$.

[3] W. H. Guier and G. C. Weiffenbach, Theoretical analysis of Doppler radio signals from earth satellites, Nature 181 (1958), no. 4622, 1525-1526.

[4] P. Kaufmann and A. Costa da Silveira, Geolocal: new space telecommunication concept for remote positioning and navigation, Proceedings of the International Workshop on Telecommunications (IWT '04), Minas Gerais, August 2004, pp. 69-72.

[5] P. Kaufmann and Fundação Instituto Nacional de Telecomunicações, Geographic and space positioning system and process, Brazil Patent PI 03003968-4, filed 17 May 2002; International Patent Cooperation Treaty PCT/BR2004/000190, filed 04 November 2004.

[6] J. Kouba, A review of geodetic and geodynamic satellite Doppler positioning, Reviews of Geophysics and Space Physics 21 (1983), no. 1, 27-40.

[7] C. N. M. Marins, P. Kaufmann, P. L. Kaufmann, T. Cordaro, L. A. Beraldo, and A. Costa da Silveira, Digital ranging telecommunication network for new geopositioning system demonstration, to appear in Journal of Microwaves and Optoelectronics.

[8] Universidade de São Paulo and P. Kaufmann, Sistema e processo de posicionamento geográfico e navegação, Brazil Patent PI 910270, granted September 1997, filed March 1991, and Addition Certificate CI 9101270, filed May 2002. 
[9] R. Vilhena de Moraes, P. L. Kaufmann, H. K. Kuga, L. A. Beraldo, P. Kaufmann, and C. N. M. Marins, Orbit determination errors in a new concept for geopositioning and navigation, 36th COSPAR Scientific Assembly, Beijing, 2006, (submitted to the ASR Journal).

[10] D. Wells (ed.), Guide to GPS Positioning, Canadian GPS Association, New Brunswick, 1987.

P. L. Kaufmann: Instituto de Matemática e Estatística, Universidade de São Paulo, 05508-090 São Paulo, SP, Brazil

E-mail address: plkaufmann@yahoo.com.br

R. Vilhena de Moraes: Faculdade de Engenharia de Guaratinguetá, Universidade Estadual de São Paulo, 012516-010 Guaratinguetá, SP, Brazil

E-mail address: rodolpho@feg.unesp.br

H. K. Kuga: Divisão de Mecânica Espacial e Controle, Instituto Nacional de Pesquisas Espaciais, 12227-010 São José dos Campos, SP, Brazil

E-mail address: hkk@dem.inpe.br

L. A. Beraldo: Instituto Nacional de Telecomunicações, 37540-000 Santa Rita do Sapucaí, MG, Brazil E-mail address: luciano-beraldo@inatel.br

C. N. Motta Marins: Instituto Nacional de Telecomunicações, 37540-000 Santa Rita do Sapucaí, MG, Brazil

E-mail address: carlosn@inatel.br

P. Kaufmann: Centro de Radioastronomia e Astrofísica Mackenzie, Universidade Presbiteriana

Mackenzie, 1302-907 São Paulo, SP, Brazil; Instituto Nacional de Telecomunicações, 37540-000 Santa Rita do Sapucaí, MG, Brazil

E-mail address: pierre.kaufmann@pesquisador.cnpq.br 


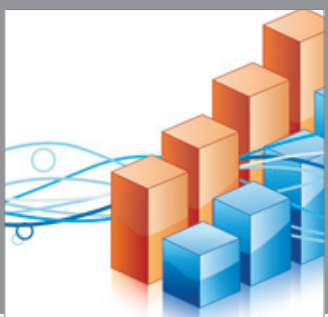

Advances in

Operations Research

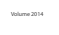

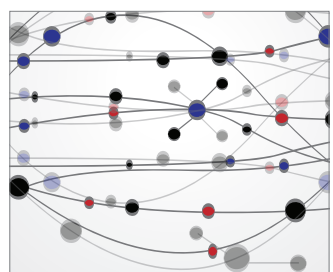

\section{The Scientific} World Journal
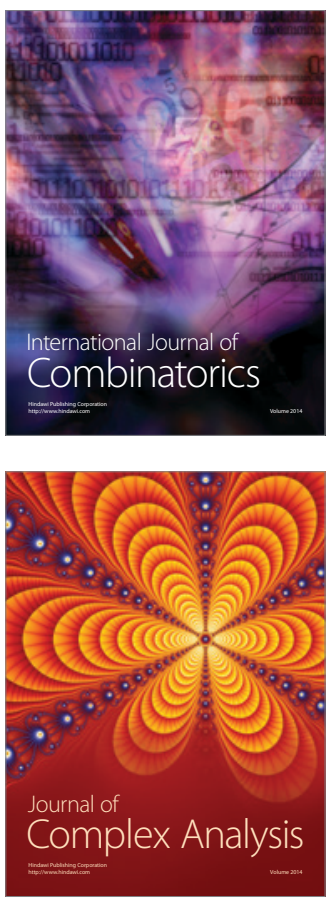

International Journal of

Mathematics and

Mathematical

Sciences
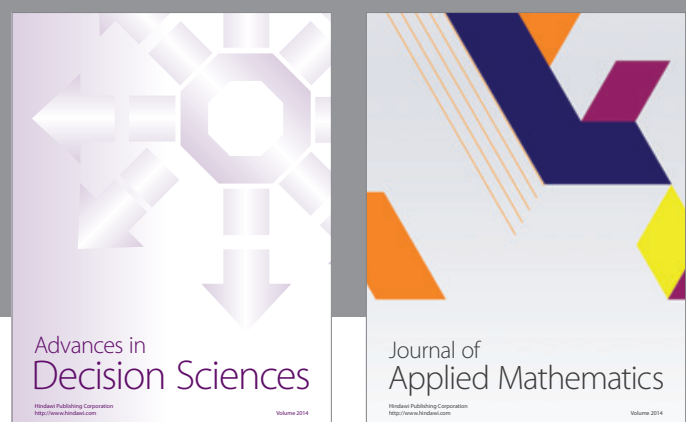

Journal of

Applied Mathematics
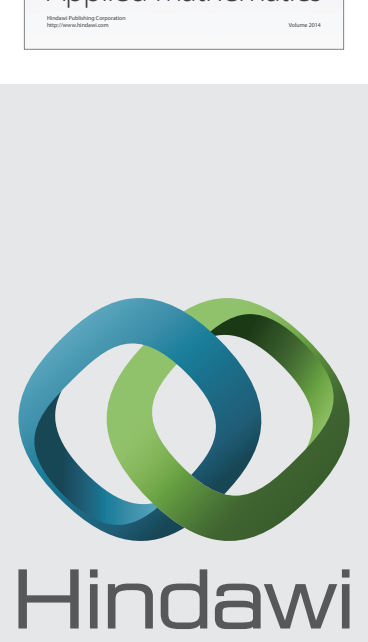

Submit your manuscripts at http://www.hindawi.com
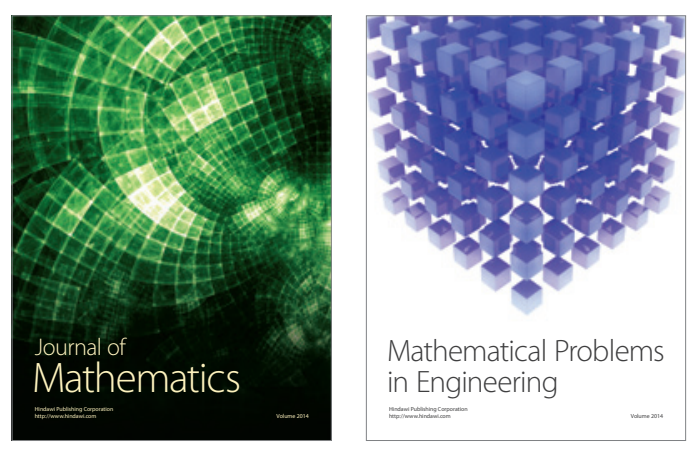

Mathematical Problems in Engineering
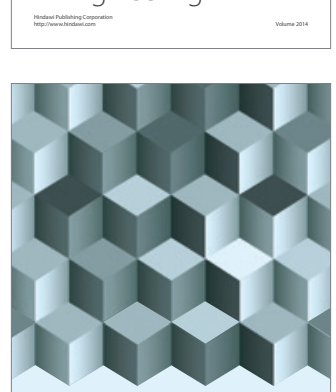

Journal of

Function Spaces
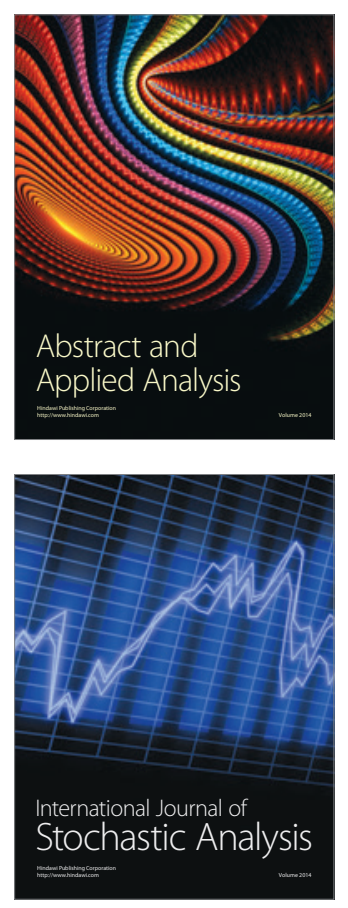

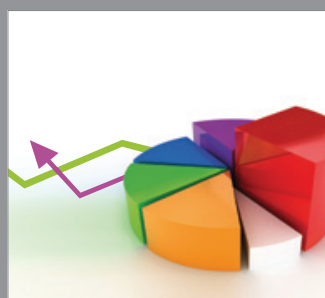

ournal of

Probability and Statistics

Promensencen
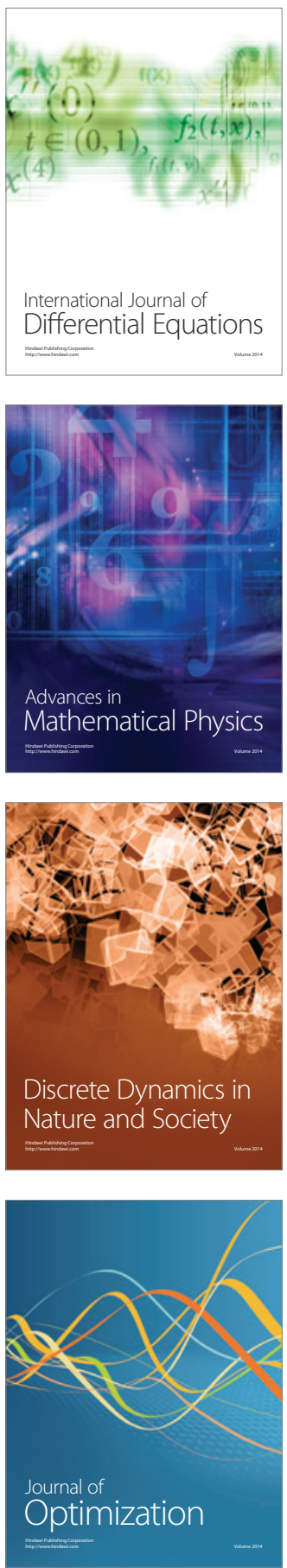